\title{
Comparison of the Colonization Ability of Autochthonous and Allochthonous Strains of Lactobacilli in the Human Gastrointestinal Tract
}

\author{
Steven A. Frese, Robert W. Hutkins, Jens Walter* \\ Department of Food Science and Technology, University of Nebraska, Lincoln, USA

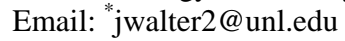

Received July 3, 2012; revised August 8, 2012; accepted August 17, 2012

\begin{abstract}
Bacteria of the genus Lactobacillus are widely used as oral probiotics due to their putative health benefits. In this study, we compared the colonization ability of two Lactobacillus strains that were identified as autochthonous to the human gastrointestinal tract (Lactobacillus reuteri ATCC PTA 6475 (MM4-1a) and Lactobacillus mucosae FSL-04) with that of an allochthonous strain (Lactobacillus acidophilus DDS-1). Colonization ability was tested in a single-blinded, cross-over study, with twelve human subjects. The test strains were quantified in fecal samples by two independent methods, selective plating and molecular typing and quantitative real time PCR. The study revealed that the two autochthonous strains (L. reuteri ATCC PTA 6475 and L. mucosae FSL-04) reached higher population levels in fecal samples and were recovered more frequently from subjects compared to the allochthonous strain (L. acidophilus DDS-1). All three strains became undetectable 8 days after the end of consumption with one exception, showing that persistence of all three strains remains short term in most individuals. In conclusion, this study showed that autochthonous Lactobacillus strains can be established more efficiently, albeit temporarily, in the human gastrointestinal tract, suggesting that evolutionary and ecological characteristics could be valuable criteria for the selection of probiotic strains.
\end{abstract}

Keywords: Lactobacillus; Probiotic; Lactobacillus reuteri; Lactobacillus mucosae; Lactobacillus acidophilus; Gut Microbiology; Microbial Ecology; Colonization Resistance

\section{Introduction}

Probiotic bacteria are defined by the FAO/WHO "as live organisms which when administered in adequate amounts confer a health benefit to the host." Implied in this definition is the expectation that these orally ingested organisms would reach the intestinal tract alive and at physiologically relevant levels [1]. Probiotic microorganisms are hypothesized to be functionally active in the human gut, such that they can influence the host through a variety of physiological mechanisms, including direct effects on the host immune system, in situ production of bioactive compounds, and competition with the resident microbiota and pathogens [2]. Some probiotic functions, such as in vivo production of bioactive compounds and competition with pathogens, also require that probiotic bacteria are metabolically active in the human gut and competitive under the prevailing conditions. Accordingly, it is often considered an important prerequisite of probiotic cultures that they originate from humans in order to

${ }^{*}$ Corresponding author. ensure adaptation and persistence in the human gut [1, 3,4].

Although many of the commercially available probiotic strains are of human fecal origin and are capable of surviving gastrointestinal passage, they are still rapidly eliminated after administration has ended [5-12]. The inability of probiotic bacteria to persist in the human intestinal tract has been attributed to the phenomenon of colonization resistance, whereby the resident gut microbiota restricts access of allochthonous organisms [13]. Moreover, even strains that are true autochthonous members of the microbiota of specific human subjects may not be able to colonize the gastrointestinal tract (GIT) of other humans due to individual differences [14].

However, it is also important to recognize that many strains currently used as probiotics belong to species which are considered allochthonous to the human intestinal tract $[14,15]$. Species often used as probiotics such as Lactobacillus acidophilus, Lactobacillus casei, Lactobacillus paracasei, Lactobacillus rhamnosus, Lactobacillus delbrueckii, Lactobacillus brevis, Lactobacillus 
johnsonii, Lactobacillus plantarum, and Lactobacillus fermentum, although commonly found in fecal samples, have never been shown to form stable populations in the human gut and are likely to originate from food or the oral cavity [14,16]. In contrast, other species of Lactobacillus, in particular, Lactobacillus reuteri, Lactobacillus ruminis, Lactobacillus gasseri, and Lactobacillus salivarius, have been reported to be autochthonous to the human gastrointestinal tract $[15,16]$. As autochthonous members of the gut microbiota, these species are likely to occupy specific niches that allow their replication and the establishment of stable population over long periods [17]. Recent comparative genome studies have begun to characterize the molecular basis of autochthony in the species L. gasseri, $L$. reuteri, and L. ruminis, and these studies identified adaptive traits that might contribute to the ecological success of lactobacilli in the human GIT [1820].

In this study, our goal was to compare survival and persistence rates of orally-consumed autochthonous and allochthonous Lactobacillus strains in human subjects. Three strains were included in this study, $L$. reuteri ATCC PTA 6475 (MM4-1a), L. mucosae FSL-04, and $L$. acidophilus DDS1. L. reuteri is a species long considered to be an autochthonous member of the human gut microbiota [16]. Strain ATCC PTA 6475 is a member of the MLSA lineage II of L. reuteri, which is almost completely composed of strains of human fecal origin, indicating that this subpopulation is adapted to the human GIT [21]. The second strain, L. mucosae FSL-04, was continuously isolated from fecal samples from a single healthy adult subject in high numbers during a 15-week period (see below). The third strain, L. acidophilus DDS-1, is a commonly-consumed probiotic $[4,22]$ belonging to the species $L$. acidophilus which is considered allochthonous to the human GIT [14-16,23,24]. To com- pare establishment and persistence of these three strains in the human gastrointestinal tract, we performed a human cross-over study in which healthy individuals consumed the test strain and provided fecal samples that were then analyzed by cultural enumeration, molecular typing of isolates, and quantitative real-time PCR (qPCR).

\section{Materials and Methods}

\subsection{Use of Human Subjects}

The human trial of this study was approved by the Institutional Review Board of the University of Nebraska (IRB Approval Number: 2009079919FB), and written informed consent has been obtained from all subjects.

\subsection{Bacterial Strains and Culture Conditions}

Strains used in this study are listed in Table 1. All strains were grown in Mann-Rogosa-Sharpe (MRS) (Difco) supplemented with $1.0 \%$ Maltose and $0.5 \%$ Fructose under anaerobic conditions at $37^{\circ} \mathrm{C}$. L. acidophilus DDS-1 was provided by Nebraska Cultures (Walnut Creek, CA USA). L. reuteri ATCC PTA 6475 (MM4-1a) was obtained from BioGaia (Stockholm, Sweden). L. mucosae FSL-04 was isolated from the feces of a healthy adult human during a previous human trial as described below [29].

\subsection{Molecular Typing and Identification of Isolates}

The molecular typing of strains was performed by Random Amplification of Polymorphic DNA (RAPD) using the primer M13V (Table 2) as described by Meroth et al. [32]. Isolates were assigned to species by 16S rRNA gene comparisons as described by Hammons et al. [33]. To obtain around $1300 \mathrm{bp}$ of the 16S rRNA gene for ex-

Table 1. Strains used in this study.

\begin{tabular}{lll}
\hline Strain & Origin & Reference or source \\
\hline L. reuteri ATCC PTA 6475 (MM4-1A) & Human & BioGaia AB (Stockholm, Sweden) \\
L. reuteri 100-23 & Rat & [25] \\
L. reuteri mlc3 & Mouse & [21] \\
L. reuteri lpuph & Mouse & This study \\
L. mucosae FSL-04 & Human, fecal sample & [26] \\
L. mucosae S5 (DSM 13346) & Porcine, small intestine & [27] \\
L. mucosae 1028 & Porcine, small intestine & [27] \\
L. mucosae 1031 & Porcine, small intestine & Nebraska cultures (Walnut Creek, CA) \\
L. acidophilus DDS-1 & Milk & [28] \\
L. acidophilus ATCC 4356 & Human &
\end{tabular}


Table 2. Primers used in this study.

\begin{tabular}{|c|c|c|c|}
\hline Primer name & Sequence 5' - > 3' & Target & Reference \\
\hline $16 \mathrm{~S} / \mathrm{p} 2$ & CTTGTACACACCGCCCGTC & 1388-1406 16S rRNA & {$[30]$} \\
\hline 23S/p10 & ССТTTCCСTCACGGTACTG & 546-474 23S rRNA & {$[30]$} \\
\hline F_acidophilus_IS & GAAAGAGCCCAAACCAAGTGATT & 16S-23S rDNA spacer & {$[31]$} \\
\hline R_acidophilus_IS & CTTCCCAGATAATTCAACTATCGCTTA & 16S-23S rDNA spacer & {$[31]$} \\
\hline L.mucosae_For & CACAATTAAACCGAGAACACC & 16S-23S rDNA spacer & This Study \\
\hline L. mucosae_Rev & ATGATCTTACGATCACCTCAGTTA & 16S-23S rDNA spacer & This Study \\
\hline L. reuteri_For & AACAATAAACCGAGAACACC & 16S-23S rDNA spacer & This Study \\
\hline L. reuteri_Rev & ССТТСАТААСТТААССТАААСАА & 16S-23S rDNA spacer & This Study \\
\hline M13V & GTTTCCCCAGTCACGAC & -- & \\
\hline
\end{tabular}

act classification, PCR products were generated and sequenced with primers 8F (5'-AGAGTTTGATCCTGGCTCAG-3') and 1391R (5'-GACGGGCGGTGWGTRCA-3’).

\subsection{Preparation of Lactobacillus Doses}

Food-grade freeze-dried powders of $L$. reuteri ATCC PTA 6475, L. mucosae FSL-04, and L. acidophilus DDS-1 were prepared by Culture Systems, Inc (Mishawaka, Indiana USA) and stored at $-20^{\circ} \mathrm{C}$ throughout the study. Upon receipt, viable cell counts and purity of all three preparations were determined and strain identity was verified by RAPD. Prior to each feeding period, viable cell counts were determined in the powders to adjust the daily dose of $10^{9}$ cells, for each strain, where necessary. Subjects were provided with pre-dosed freeze-dried preparations of lactobacilli and instructed to store the preparations at $4^{\circ} \mathrm{C}$ and reconstitute the powder in $40 \mathrm{~mL}$ cold or room-temperature milk and consume at their own convenience, with a meal.

\subsection{Human Trial}

A blinded, crossover study was performed with twelve healthy adult humans (six male and six female, age 21 27). Subjects were selected to be tolerant of milk products, free of chronic gastrointestinal disorders, nonvegetarians, and had not consumed antibiotics in the two months prior to the study. No dietary restrictions were placed on participants, except to avoid probiotic supplements, cultured dairy products, or products advertised as having "live and active cultures". The study was conducted over three separate 8-week periods (each period separated by 3 to 4 weeks) where the individual strains were tested in succession. Each feeding period began with a two-week baseline period (no change in diet). The subjects then consumed a daily dose of $10^{9}$ viable bacte- rial cells for 7 days, followed by a 5 week wash out period. Fecal samples were collected weekly, resulting in 2 fecal samples during the baseline period, and fecal samples taken at day 1,8 , and 15 of the wash out periods. The human trial of this study was approved by the Institutional Review Board of the University of Nebraska (IRB Approval Number: 20090-79919FB), and written informed consent was obtained from all subjects.

Subjects completed a symptoms diary to assess the potential side effects of experimental strain administration. The symptoms included were bowel movement, stool consistency, discomfort, flatulence, abdominal pain, and bloating, and subjects were asked to score them on a scale from 1 (none, normal, good well-being) to 5 (severe symptoms and discomfort). All twelve subjects completed the trials, and self-reported compliance with the experimental treatments was $100 \%$.

\subsection{Microbial Analysis of Fecal Samples}

Subjects provided fresh fecal samples in sterile fecal sample collection containers, and samples were processed within four hours of defecation. A ten-fold dilution of each sample in sterile phosphate buffered saline (PBS) (pH 7.0) was immediately frozen at $-80^{\circ} \mathrm{C}$ for later DNA extraction (see below). Furthermore, a 10-fold dilution series was made with sterile saline $(0.9 \% \mathrm{NaCl})$, and aliquots were plated on Rogosa SL Agar, which is selective for lactobacilli.

Plates were incubated anaerobically for 48 hours at $37^{\circ} \mathrm{C}$ before enumeration. To analyze the total Lactobacillus population, 10 colonies were picked at random from a dilution agar plate containing about 100 colonies. Selection of colonies was randomized by drawing intersecting lines across the plate, and picking colonies along the lines until ten had been recovered, in order to remove operator bias. The isolates were differentiated after subculture by RAPD analysis through direct comparison 
with the molecular fingerprint obtained with the test strains (Figure 1), a strategy which has been effectively used to differentiate Lactobacillus isolates from oral and fecal samples $[33,34]$.

To determine the bacterial population of the test strains in fecal samples, total counts of lactobacilli (on Rogosa SL Agar) were multiplied by the percentage of the strain among the 10 typed colonies.

Although this analysis would under-estimate the numbers of the test strains in fecal samples from subjects with background Lactobacillus counts, the analysis still allowed the detection of a significant increase due to the administration of the strain during the test period.

\subsection{Quantitative Real-Time PCR (qPCR)}

Lactobacillus strains were quantified in human fecal samples by qPCR. Strain specific PCR systems could not be designed for the human $L$. reuteri strains of the MLSA lineage II and for L. acidophilus strains, as these strains are clonal at all loci that were tested [20]. Therefore, species-specific primers that targeted the 16S-23S rDNA intergenic spacer region (Table 2) were used. Specific primers for $L$. acidophilus targeting this region were previously described by Haarman and Knol [31]. Primers for $L$. reuteri and $L$. mucosae were designed to also target the same region within the 16S-23S rDNA spacer. For $L$. mucosae, the 16S-23S rDNA spacer region was amplified from four strains (Table 1) by primers 16S/p2 and 23S/p10 [30] (Table 2) and sequenced (Table 3). Sequences for $L$. reuteri were obtained from available ge-

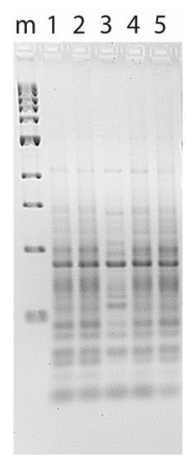

(a)

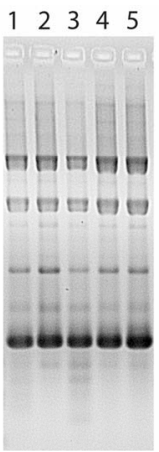

(b)

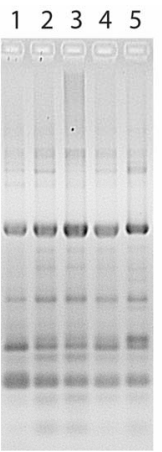

(c)

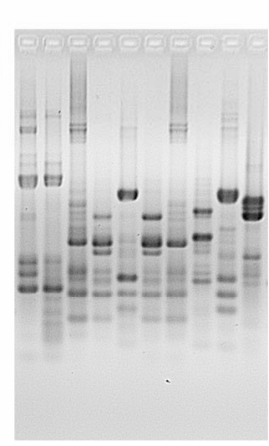

(d)
Figure 1. Identification of Lactobacillus strains by RAPD typing. RAPD patterns from $L$. reuteri MM4-1a (a); L. mucosae FSL-04 (b); and L. acidophilus DDS-1 (c). Each lane represents a RAPD-typed culture obtained from the original stock culture (lane 1), the freeze-dried powders used in the human trial (lane 2), and from colonies isolated from subject fecal samples after consumption of the strain (lane 3 - 5). Examples of isolates obtained by fecal culture during this study from multiple subjects indicating banding patterns are distinct between strains (d). Lane m: $1 \mathrm{~kb}$ DNA ladder (New England Biolabs, Massachusetts USA). nome sequences (L. reuteri ATCC PTA 6475, 100-23, mlc3, and lpuph), and sequences were aligned using CLUSTALW to generate consensus sequences from which species-specific primers were constructed. Primers were validated for target specificity using DNA from lactobacilli-negative fecal samples $\left(<10^{2} \mathrm{CFU} / \mathrm{g}\right.$ lactobacilli) and DNA isolated from strains of each of the species of Lactobacillus used (Table 1).

Specificity for all primers was further validated in silicio using the NCBI database.

Genomic DNA was extracted from fecal samples as described previously [24]. qPCR was performed in a Mastercycler Realplex2 (Eppendorf AG, Hamburg, Germany) using the Quanti-Fast SYBR Green PCR system (Qiagen, Düsseldorf, Germany) as directed by the manufacturer. The PCR program consisted of a single $95^{\circ} \mathrm{C}$ step for 5 mins, followed by 40 cycles of a two-step PCR reaction, beginning with a 10 -second $95^{\circ} \mathrm{C}$ denaturation step and a 30 second $60^{\circ} \mathrm{C}$ annealing/extension step. Melting curves were also performed, consisting of a denaturation step of $15 \mathrm{~s}$ at $95^{\circ} \mathrm{C}$, an increase from $60^{\circ} \mathrm{C}$ $95^{\circ} \mathrm{C}$ over a 20 -min period, and a final step of $15 \mathrm{~s}$ at $95^{\circ} \mathrm{C}$. Reactions were performed in $25 \mu \mathrm{L}$ volumes containing $0.5 \mu \mathrm{m}$ each primer and $1 \mu \mathrm{L}$ of extracted DNA.

Standard curves for absolute quantification were prepared from overnight cultures (14 h) of each organism ( $L$. reuteri ATCC PTA 6475, L. mucosae FSL-04, and $L$. acidophilus DDS-1) that were plated in triplicate and enumerated, in parallel to DNA extraction from $1 \mathrm{ml}$ of the culture. A tenfold dilution series was generated with this DNA in $10 \mathrm{mM}$ Tris- $\mathrm{Cl} \mathrm{pH} 8.0$ to generate a range of DNA concentrations representing $10^{9}$ cells to $10^{4}$ cells, based on culture-based enumeration from the original culture. Reactions were performed in triplicate for the standard curves and in duplicate for fecal DNA samples. The correlation coefficient, $\mathrm{r}^{2}$, for the three standard

Table 3. Sequences determined during this study.

\begin{tabular}{lll}
\hline Strain & Sequence determined & Accession number \\
\hline $\begin{array}{l}\text { L. mucosae } \\
\text { FSL-04 }\end{array}$ & $\begin{array}{l}\text { 16S/23S intergenic } \\
\text { spacer region }\end{array}$ & JN368428 \\
& Partial 16S rRNA gene & JN092131 \\
& $\begin{array}{l}\text { 16S/23S intergenic } \\
\text { spacer region }\end{array}$ & JN592586 \\
L. mucosae S5 & $\begin{array}{l}\text { 16S/23S intergenic } \\
\text { spacer region }\end{array}$ & JN592585 \\
L. mucosae 1028 & $\begin{array}{l}\text { 16S/23S intergenic } \\
\text { spacer region }\end{array}$ & JN592584 \\
L. mucosae 1031 & $\begin{array}{l}\text { 16S/23S intergenic } \\
\text { spacer region }\end{array}$ & JN368427 \\
L. acidophilus DDS-1 & $\begin{array}{l}\text { Partial 16S rRNA } \\
\text { gene }\end{array}$ & JN368429 \\
\hline L. acidophilus DDS-1
\end{tabular}


curves were 0.99 and PCR efficiencies were 0.93 ( $L$. mucosae), 0.98 (L. reuteri) and 1.00 (L. acidophilus).

The qPCR systems were validated by spiking a lactobacilli-negative fecal sample (less than $10^{2} \mathrm{CFU}$ per gram lactobacilli as determined by selective culture on Rogosa SL Agar) with 10 to $10^{8}$ cells per gram of each of the three test strains. DNA was extracted and qPCR was performed in duplicate as described above. Predicted numbers of lactobacilli demonstrated a linear dynamic range closely reflecting the number of known added cells $\left(r^{2}>0.98\right.$ for MM4-1a, 0.99 for FSL-04, and 0.93 for DDS-1) from $10^{4}$ cells per g to $10^{8} \mathrm{CFU}$ per gram. Below $10^{4}$ cells per gram fecal samples, background signal prevented accurate quantification. Within the dynamic range, cell numbers determined by qPCR were in agreement with the cell numbers that were spiked to the samples as indicated by the slopes of the trend line in linear regression ( $b=0.91$ for ATCC PTA 6475, $b=0.86$ for FSL-04 and $b=1.01$ for DDS-1) when predicted cell numbers were plotted against cells added.

\subsection{Statistical Analysis}

Results are presented as means \pm standard deviations. Statistical tests for treatment effects of test strain administration on the abundance of individual strains or abundance of Lactobacillus species were performed either by one-way analysis of variance (ANOVA) with repeated measure or by one-way ANOVA, followed by Tukey post-hoc test.

\section{Results}

\subsection{Selection of Lactobacillus mucosae FSL-04 as an Autochthonous Member of the Human Intestinal Tract}

To identify human autochthonous lactobacilli, we screened fecal samples from 11 human subjects that had participated in a previous study [29]. Fecal Lactobacillus populations were quantified over the duration of four months by plating serial dilutions on Rogosa SL Agar plates. As observed in previous studies [14,15,24,34], most of the subjects harbored low numbers of lactobacilli in fecal samples (less than $10^{6} \mathrm{CFU} / \mathrm{g}$ ). However, one subject consistently shed high levels of lactobacilli in fecal samples (Figure 2(a)) over the entire 4 month study. Two dominant colony types were identified, and 28 isolates were picked randomly during the 15 week duration of the trial representing both colony morphologies.

Molecular typing of these isolates by RAPD revealed the presence of two unique strains. One strain was detected throughout the entire 15 week time period by RAPD-typing (Figure 2(b)) and classified as L. mucosae by 16S rRNA sequence analysis (>99.9\% homology to 16S rRNA gene from L. mucosae CCUG 43179T). Given that this strain was found in the fecal samples of this human subject in high numbers, we concluded that it represented an autochthonous member of the gut microbiota.
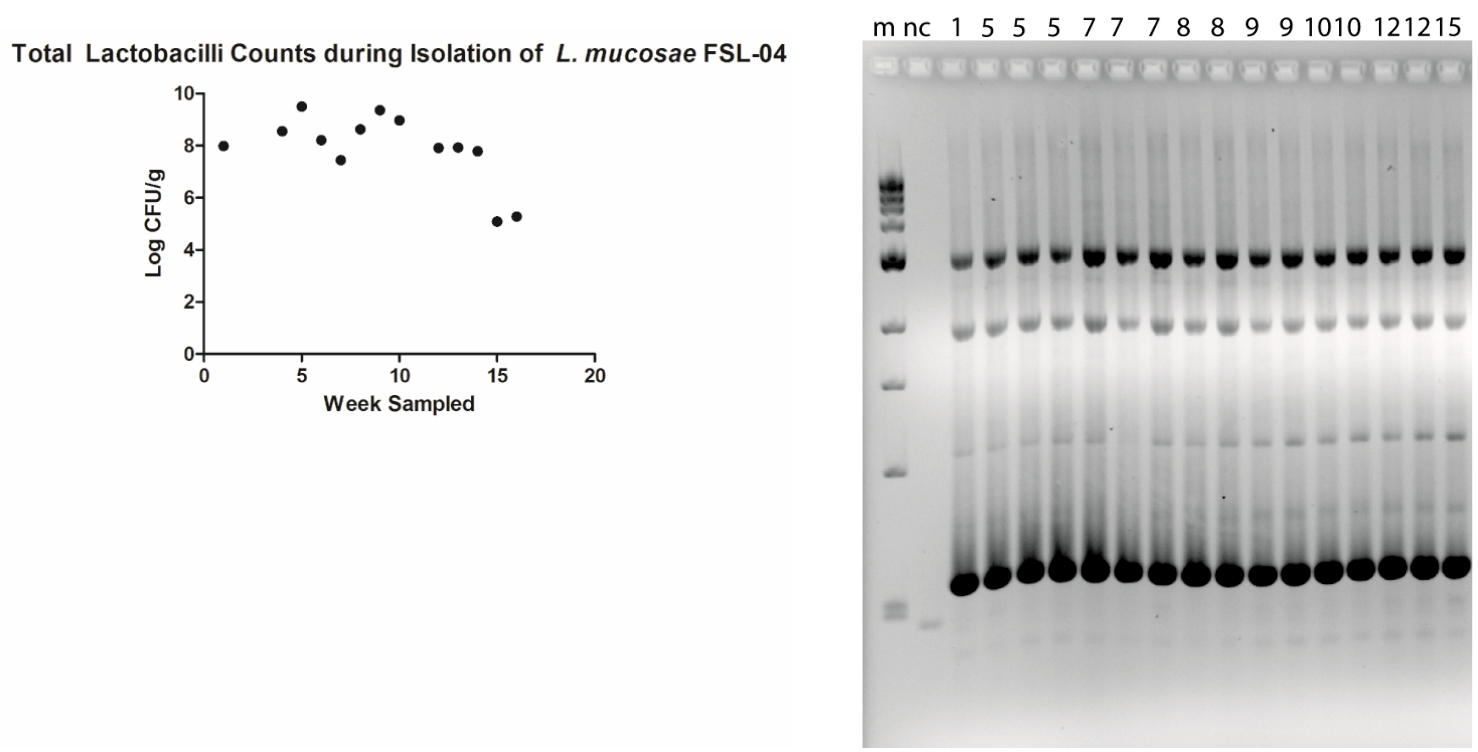

(a)

(b)

Figure 2. Identification of Lactobacillus mucosae FSL-04 as an autochthonous member of the human gut microbiota. (a) Total Lactobacilli (CFU/gram feces) enumerated from Rogosa SL agar over several weeks during isolation of FSL-04 from a single healthy adult human; (b) RAPD patterns of isolates identified as Lactobacillus mucosae obtained from these fecal samples. Lane m: 1kb DNA ladder (New England Biolabs, Massachusetts USA); Lane nc: Negative control. Numbered lanes correspond to the respective weeks isolates were obtained. 
Importantly, no lactobacilli were cultured from saliva samples obtained from this subject at three different time points (data not shown), excluding the oral cavity as a potential origin of this strain. A single isolate (L. mucosae FSL-04) was selected for use in this study.

\subsection{Human Trial to Compare Persistence of Lactobacillus Strains}

We performed a human cross-over feeding study to compare the intestinal establishment and persistence of $L$. reuteri ATCC PTA 6475, L. mucosae FSL-04, and $L$. acidophilus DDS-1 after oral administration of a daily dose containing viable cells for 7 days to 12 human subjects. No adverse effects, as assessed by symptoms diaries querying bowel movement, stool consistency, general discomfort, flatulence, abdominal pain, and bloating, were detected for any of the strains during the feeding period (data not shown). The Lactobacillus population was characterized in fecal samples two weeks before subjects received the probiotic strains. Persistence was tested at day 1, 8 and 15 of the post-test period by quantitative culture and qPCR. The proportion of each probiotic strain as a percent of the total cultivable Lactobacillus population was determined by RAPD typing of 10 random isolates at each time-point, a number of isolates that has been shown to give a sufficient overview about the Lactobacillus strain distribution in human fecal samples [15]. As shown in Figure 1, each strain had a distinct RAPD-pattern and could easily be distinguished from other isolates obtained throughout the study.

\subsection{Transient Recovery of Lactobacilli from Humans after Administration of Three Test Strains}

The number of fecal lactobacilli varied markedly among the 12 subjects during the baseline period, ranging from $<10^{2}$ to $10^{9} \mathrm{CFU} / g r a m$, with an average of around $10^{4}$ CFU/gram (Table 4). RAPD analysis revealed that none

Table 4. $\log _{10}$ total lactobacilli populations in subject fecal samples before (baseline 1 and 2 ) and post-test (Day 1, 8, and 15). Percentages of isolates typed as the respective probiotic strain by RAPD are shown in parentheses (nd, not determined).

\begin{tabular}{|c|c|c|c|c|c|c|c|c|c|c|c|c|c|}
\hline \multicolumn{14}{|c|}{ Total Lactobacilli in $\log _{10}$ CFU/g Feces (percent of isolates typed as the probiotic strain) } \\
\hline \multicolumn{14}{|c|}{ Subject } \\
\hline Strain & 1 & 2 & 3 & 4 & 5 & 6 & 7 & 8 & 9 & 10 & 11 & 12 & Mean \\
\hline \multicolumn{14}{|l|}{ Baseline 1} \\
\hline $\begin{array}{l}\text { ATCC PTA } \\
6475\end{array}$ & 8.64 (nd) & $<2$ (nd) & $<2$ (nd) & $<2$ (nd) & $<2$ (nd) & 2.77 (nd) & 3.29 (nd) & 3.47 (nd) & $<2$ (nd) & $<2$ (nd) & 3.57 (nd) & 4.21 (nd) & 3.16 (nd) \\
\hline FSL-04 & 4.30 (nd) & 3.20 (nd) & 3.00 (nd) & 5.85 (nd) & 5.30 (nd) & 5.76 (nd) & $<2$ (nd) & 6.79 (nd) & $<2$ (nd) & $<2$ (nd) & 4.43 (nd) & 2.84 (nd) & 3.96 (nd) \\
\hline DDS $<21$ & 2.60 (nd) & 2.30 (nd) & 5.95 (nd) & 7.59 (nd) & $<2$ (nd) & 5.68 (nd) & 3.55 (nd) & 3.00 (nd) & $<2$ (nd) & $<2$ (nd) & 4.65 (nd) & $<2$ (nd) & 3.61 (nd) \\
\hline \multicolumn{14}{|l|}{ Baseline 2} \\
\hline $\begin{array}{l}\text { ATCC PTA } \\
6475\end{array}$ & $<2(0)$ & $6.39(0)$ & $<2(0)$ & $6.94(0)$ & $<2(0)$ & $3.96(0)$ & $3.55(0)$ & $<2(0)$ & $<2(0)$ & $<2(0)$ & $<2(0)$ & $3.30(0)$ & $3.18(0)$ \\
\hline FSL-04 & $5.84(0)$ & $<2(0)$ & $6.82(0)$ & $7.92(0)$ & $<2(0)$ & $4.70(0)$ & $4.75(0)$ & $3.41(0)$ & $4.63(0)$ & $<2(0)$ & $4.19(0)$ & $<2(0)$ & $4.19(0)$ \\
\hline DDS-1 & $5.40(0)$ & $4.74(0)$ & $6.30(0)$ & $7.16(0)$ & $6.48(0)$ & $2.30(0)$ & $3.30(0)$ & $2.30(0)$ & $<2(0)$ & $<2(0)$ & $3.58(0)$ & $4.77(0)$ & $4.19(0)$ \\
\hline \multicolumn{14}{|l|}{ Day 1} \\
\hline $\begin{array}{l}\text { ATCC PTA } \\
6475\end{array}$ & 7.37 (100) & $6.79(90)$ & $6.45(100)$ & $7.46(100)$ & 5.09 (100) & $4.34(90)$ & $3.51(80)$ & $6.21(0)$ & 3.57 (100) & 3.90 & $4.44(90)$ & $5.03(60)$ & 5.35 \\
\hline FSL-04 & $4.81(100)$ & $6.17(40)$ & $3.49(0)$ & $8.01(10)$ & $5.72(70)$ & $6.11(40)$ & $5.34(90)$ & $4.97(80)$ & $5.16(90)$ & $3.43(100)$ & $5.20(0)$ & $5.21(90)$ & 5.30 (59) \\
\hline DDS-1 & $3.83(0)$ & 4.87 (100) & $5.01(20)$ & $4.38(0)$ & $5.09(90)$ & $7.09(0)$ & $5.06(70)$ & $5.56(10)$ & $<2(0)$ & $4.52(0)$ & $5.13(0)$ & $5.76(0)$ & $4.86(24)$ \\
\hline \multicolumn{14}{|l|}{ Day 8} \\
\hline $\begin{array}{l}\text { ATCC PTA } \\
6475\end{array}$ & $4.90(0)$ & $<2(0)$ & $4.34(100)$ & $5.77(0)$ & $<2(0)$ & $<2(0)$ & $3.27(0)$ & $5.30(0)$ & $<2(0)$ & $<2(0)$ & $<2(0)$ & $4.95(0)$ & 3.38 (8) \\
\hline FSL-04 & $3.94(0)$ & $2.90(0)$ & $<2(0)$ & $7.15(0)$ & $2.90(0)$ & $3.77(0)$ & $2.84(0)$ & $4.89(0)$ & $5.05(0)$ & $2.47(0)$ & $4.41(0)$ & $4.05(0)$ & $3.86(0)$ \\
\hline DDS-1 & $4.22(0)$ & $<2(0)$ & $<2(0)$ & $8.18(0)$ & $2.47(0)$ & $5.28(0)$ & $<2(0)$ & $2.60(0)$ & $4.43(0)$ & $<2(0)$ & $4.03(0)$ & $<2(0)$ & $3.43(0)$ \\
\hline \multicolumn{14}{|l|}{ Day 15} \\
\hline $\begin{array}{l}\text { ATCC PTA } \\
6475\end{array}$ & $4.54(0)$ & $<2(0)$ & $6.41(40)$ & $6.93(0)$ & $3.30(0)$ & $3.77(0)$ & $<2(0)$ & $3.82(0)$ & $4.73(0)$ & $<2(0)$ & $<2(0)$ & $4.03(0)$ & 3.79 (3) \\
\hline FSL-04 & $<2(0)$ & $3.98(0)$ & $3.64(0)$ & $7.58(0)$ & $<2(0)$ & $5.38(0)$ & $<2(0)$ & $3.68(0)$ & $4.47(0)$ & $<2(0)$ & $6.93(0)$ & $4.13(0)$ & $3.98(0)$ \\
\hline DDS-1 & $2.84(0)$ & $2.47(0)$ & $4.03(0)$ & $7.51(0)$ & $<2(0)$ & $5.38(0)$ & $7.19(0)$ & $<2(0)$ & $4.25(0)$ & $<2(0)$ & $3.21(0)$ & $6.03(0)$ & $4.08(0)$ \\
\hline
\end{tabular}


of the three test strains were detectable during the baseline. After subjects had consumed the test strains for 7 days, culture analysis of day 1 fecal samples showed an increase in total Lactobacillus numbers to about $10^{5}$ CFU/gram. The RAPD analysis of random isolates revealed that the test strains could be detected in a majority of the subjects (Table 4), indicating that the increase in total lactobacilli detected soon after consumption was a result of administration of the respective experimental strain.

We next estimated the fecal populations of the test strains by multiplying total lactobacilli counts by the relative proportions of each strain, as determined by RAPD analysis (see Materials and Methods). Administration of $L$. reuteri ATCC PTA 6475 and L. mucosae FSL-04 led to a significant increase in the numbers of the respective strains at day 1 after consumption when compared to baseline, while administration of DDS-1 did not result in a significant increase (Figure 3(a)). The populations of L. reuteri ATCC PTA 6475 (5.22 Log $_{10}$ CFU/ gram) and L. mucosae (4.94 $\log _{10}$ CFU/gram) were higher than that of L. acidophilus (4.26 $\left.\log _{10} \mathrm{CFU} / \mathrm{gram}\right)$, but differences did not reach statistical significance.

The number of total lactobacilli returned to baseline at day 8 after consumption (Table 4 and Figure 3(a)), and the probiotic strains were not detectable anymore with one exception (L. reuteri ATCC PTA 6475 was detected at day 8 and 15 of the washout period in one subject).

\subsection{L. reuteri ATCC PTA 6475 and L. mucosae FSL-04 Can be Established More Efficiently and in Higher Numbers than L. acidophilus DDS-1}

All three test strains could be detected by the cultureRAPD method in a subset of subjects at day 1 , but the rate of recovery differed between strains (Table 4). At day 1 after administration, 84\% of the isolates were identified as L. reuteri ATCC PTA 6475 at day 1, compared to $59 \%$ and $24 \%$ for L. mucosae FSL-04 and L. acidophilus DDS-1, respectively (Figure 3(b)). The difference in recovery rate of L. reuteri ATCC PTA 6475 was significantly higher than that of L. acidophilus DDS-1 (Figure 3(b)). In addition, the autochthonous strains were detectable in fecal samples of more subjects. $L$. reuteri ATCC PTA 6475 was recovered from 11 out of the 12 subjects, L. mucosae FSL-04 and was present in 10 subjects, while $L$. acidophilus DDS-1 was present in 5 subjects.

Although the background Lactobacillus populations in human fecal samples were low, they still confounded the culture-based analyses as they prohibited an exact quantification of the test strains. To determine the establishment of the test strains in the human gastrointestinal tract, samples taken during baseline, day 1 post-test, and day 8 post-test were analyzed by species-specific qPCR. We used a species and not strain-specific primers for the qPCR, as human $L$. reuteri and $L$. acidophilus isolates are highly clonal, making the development of strain specific primers impractical. Furthermore, we chose to target the same gene (the 16S-23S rRNA spacer region) in all three strains used during this study to avoid PCR bias. Population levels were determined by absolute quantification using standard curves obtained from bacterial cells. While strain-specific primers would be advantageous, the analysis of baseline fecal samples indicated that no confounding Lactobacillus populations were present in any of the subjects. As shown in Figure 3(c), the levels of $L$. reuteri, $L$. mucosae, and $L$. acidophilus were generally below the detection limit $\left(10^{4} \mathrm{CFU} /\right.$ gram $)$ in most of the samples during baseline and at day 8 of wash-out, indicating that background levels of the species did not confound this technique.

At day 1 of wash-out, there was a statistically significant increase in each of the three species when compared to the baseline and the day 8 wash-out, with $L$. reuteri ATCC PTA 6475 reaching $5.14 \log _{10}$ cells/gram, $L$. mucosae FSL-04 reaching $5.03 \log _{10}$ cells/gram, and L. acidophilus DDS-1 $4.32 \log _{10}$ cells/gram feces. The qPCR analysis revealed that the two autochthonous strains L. reuteri ATCC PTA 6475 and L. mucosae FSL-04 reached significantly higher populations ( $\mathrm{p}<$ 0.01) in fecal samples when compared to L. acidophilus DDS-1 (Figure 3(c)).

\section{Discussion}

The objective of this study was to test whether autochthonous Lactobacillus strains (L. mucosae FSL-04 and L. reuteri ATCC PTA 6475) can be established more efficiently in the human gastrointestinal tract than an allochthonous strain (L. acidophilus DDS-1) following a 7-day feeding period. Accordingly, we characterized the Lactobacillus populations in fecal samples obtained from humans that had consumed standardized, freeze dried cell preparations of three test strains using both culture-based and molecular (qPCR) methods. As observed in previous probiotic trials, all three Lactobacillus strains survived gastric passage and were temporarily detectable in the fecal samples [5,8,12,15,35]. Most importantly, our findings indicate that the two autochthonous strains (L. reuteri ATCC PTA 6475 and L. mucosae FSL-04) reached higher populations and were generally more persistent than the allochthonous strain. In addition, the autochthonous strains, and L. reuteri ATCC PTA 6475 in particular, could be detected in a larger proportion of subjects and represented a larger percentage of the total Lactobacillus population (Table 4). 


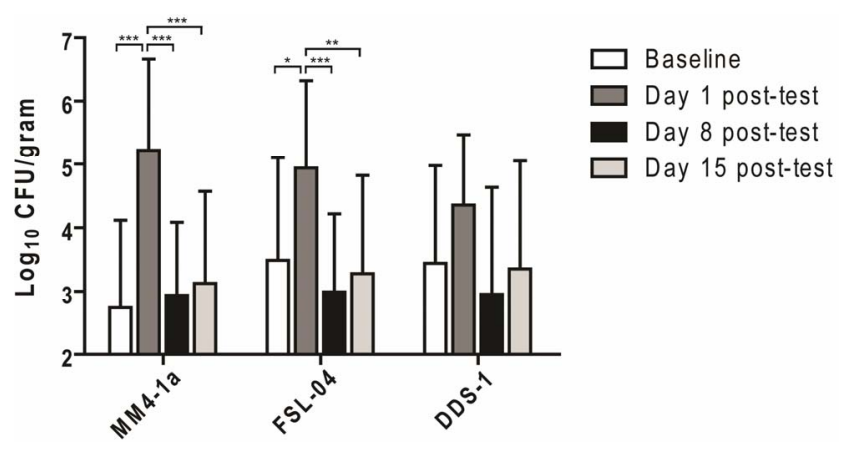

(a)

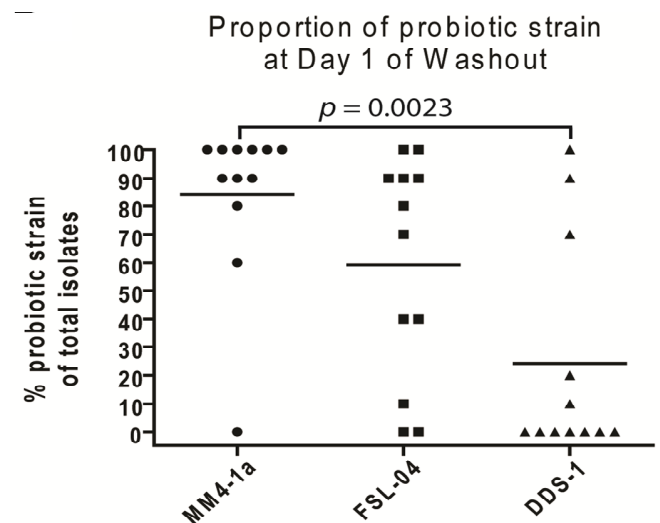

(b)

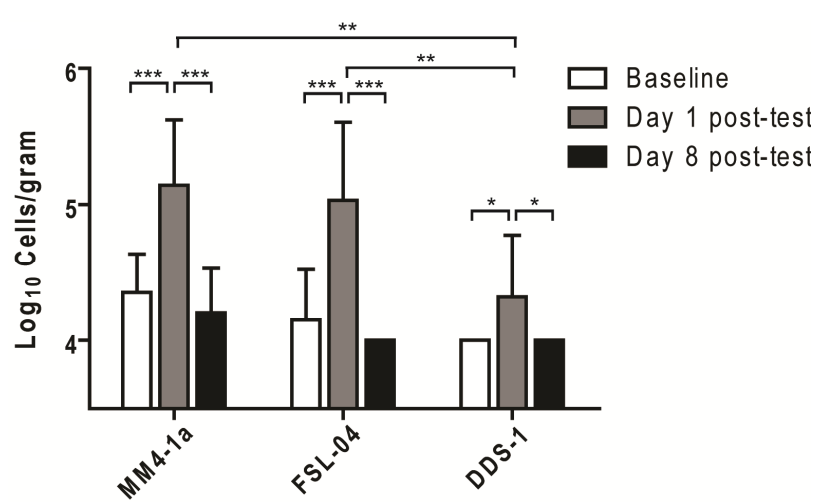

(c)

Figure 3. Quantification of probiotic strains in fecal samples of subjects as determined by culture- and molecular-based methods. (a) Culturable counts of $L$. reuteri MM4-1a, L. mucosae FSL-04, and L. acidophilus DDS-1 during baseline (second sample) and day 1,8 , and 15 post-test. Populations were determined by multiplying total number of lactobacilli with the proportion of the probiotic strain as determined by RAPD typing of 10 random colonies. Statistical analysis was performed with One-way ANOVA with repeated measures and Turkey post-hoc tests; (b) Percentage of the probiotic strains to the total Lactobacillus population at day 1 post-test as determined by RAPD typing of 10 random colonies. Statistical analysis was performed with One-way ANOVA with repeated measures and Turkey post-hoc tests; (c) Quantification of the Lactobacillus species L. reuteri (during treatment with strain MM41-1a), L. mucosae (during treatment with strain FSL-04), and L. acidophilus (during treatment with strain DDS-1) by qRT-PCR. Statistical analysis was performed with One-way ANOVA and Turkey post-hoc tests.

We suggest here that the more efficient establishment of autochthonous probiotic strains is due to their adaptation to the human gastrointestinal tract. The L. reuteri strain that was used during this study, which reached the highest levels of colonization and recovery, belongs to a subpopulation of the species that has been shown to be highly specific to the human gastrointestinal tract [21], and which possesses a genome content that reflects its adaptation to the human gastrointestinal tract [20]. $L$. mucosae is a species that is ordinarily able to bind to both mucus and human blood group antigens [26,36], and the strain used in our study was routinely detected in a human subject in high numbers over 4 months (Figure 2). It is unlikely that the population differences of $L$. reuteri and $L$. mucosae, compared to $L$. acidophilus are due to comparatively lower survival rate of $L$. acidophilus during gastric transit, as the latter species has been shown to have high rates of tolerance towards low acidity and bile acids [5]. Instead, the restricted ability of $L$. acidophilus to form stable populations in the human, as shown previously $[15,16,23,24]$ is likely due to the absence of specific adaptive features that are evidently present in L. reuteri and $L$. mucosae.

Although our data indicated that the two autochthonous Lactobacillus strains could be established in higher numbers in the human gut, the strains were no more persistent than the allochthonous strain. That probiotic bacteria can only be transiently established in the gut has been shown in many previous studies, and duration of persistence does not seem to depend on differences in inoculum dose, strain or species, carrier medium, and even duration of consumption [5,9,15]. The data obtained during our study indicates that autochthony does not increase the duration of persistence. This finding suggests that it may be difficult, if not impossible, to establish a probiotic strain long term in the gastrointestinal tract of 
most human subjects, even if that microbe was derived from a stable component of one individual's gut microbiota. Therefore, the ability of the microbiota to prevent establishment of foreign organisms (colonization resistance), applies not only to pathogens, but to other gut microbes as well.

The ecological principles that govern community assembly of the gut microbiota and determine which lineages can become established are not completely understood. Modern concepts of community ecology suggest that a combination of niche-related and historic processes (e.g. in situ evolution of early colonizers) govern the process [37-39]. Historic factors such as colonization order, transmission, and niche construction are inherently stochastic, and therefore cause variation in individual microbiomes, while in situ evolution of members ensures that members are highly adapted to available niches [39]. These concepts have important implications if the goal is to establish a probiotic organism in the gut. First, the microbiome is individualized and composed of microbes that are adapted to occupy specific niches within each person's particular community, and thus, members of microbiomes are not necessarily interchangeable. Second, establishment of new microbes is only possible as long as niches are still open (early during assembly) or if niches become available (when community members are intentionally or accidentally removed from the community). If community structure becomes disrupted, for example through a high dose of antibiotics, microbes from another individual can be successfully and more permanently established [40]. Therefore, probiotic bacteria might be more permanently established when administered after antibiotic treatments or early in life.

Although our findings indicate the autochthony of probiotic lactobacilli does not increase the duration of their persistence in the human gastrointestinal tract, the observation that autochthonous strains can be more efficiently established is clearly of practical importance. We not only detected $L$. reuteri ATCC PTA 6475 and $L$. mucosae FSL-04 in more subjects after administration, but these strains also reached about ten times higher cell numbers in fecal samples when compared to $L$. acidophilus DDS-1. These findings indicate that the effective dose of a probiotic is likely to be higher when an autochthonous strain versus an allochthonous strain is used. If probiotic action is dependent on the metabolic functionality of the organisms in the gut, such as direct antagonism or the production of bioactive substances, then a higher dose of viable organisms present the gut is likely to increase the success of a probiotic strategy. In conclusion, the results described in this study support the notion that evolutionary and ecological characteristics are valuable criteria for the selection of probiotic strains [14]. Future studies should be aimed to test persistence of other autochthonous strains, especially L. ruminis, which appears to be the dominant Lactobacillus species in the human gut $[15,16,41]$.

\section{Acknowledgements}

The dedication of the participants in the human trial is gratefully acknowledged. We are thankful to Stefan Roos (Swedish University of Agricultural Sciences) for providing L. mucosae S5, 1028, and 1031 and Dr. Todd R. Klaenhammer (North Carolina State University ) for providing L. acidophilus ATCC 4356. We thank Nebraska Cultures (Walnut Creek, CA) for providing L. acidophilus DDS-1 and partially funding the study.

\section{REFERENCES}

[1] A. C. Ouwehand, S. Salminen and E. Isolauri, "Probiotics: An Overview of Beneficial Effects," Antonie Van Leeuwenhoek, Vol. 82, No. 1-4, 2002, pp. 279-289. doi:10.1023/A:1020620607611

[2] P. W. O’Toole and J. C. Cooney, "Probiotic Bacteria Influence the Composition and Function of the Intestinal Microbiota," Interdisciplinary Perspectives on Infectious Diseases, Vol. 85, No. 1752, 2008, 9 Pages.

[3] C. Dunne, L. Murphy, S. Flynn, L. O’Mahony, S. O’Halloran, M. Feeney, D. Morrissey, G. Thornton, G. Fitzgerald, C. Daly, B. Kiely, E. M. Quigley, G. C. O’Sullivan, F. Shanahan and J. K. Collins, "Probiotics: From Myth to Reality Demonstration of Functionality in Animal Models of Disease and in Human Clinical Trials," Antonie Van Leeuwenhoek, Vol. 76, No. 1-4, 1999, pp. 279-292. doi:10.1023/A:1002065931997

[4] M. E. Sanders, "Probiotics: Considerations for Human Health,” Issue Nutrition Reviews, Vol. 61, No. 3, 2003, pp. 91-99. doi:10.1301/nr.2003.marr.91-99

[5] C. N. Jacobsen, N. Rosenfeldt, V. Hayford, A. E. Moller, P. L. Michaelsen, K. F. Paerregaard, A. Sandstrom, M. B. Tvede and M. Jakobsen, "Screening of Probiotic Activities of Forty-Seven Strains of Lactobacillus spp. by in Vitro Techniques and Evaluation of the Colonization Ability of Five Selected Strains in Humans,” Applied and Environmental Microbiology, Vol. 65, No. 11, 1999, pp. 4949-4956.

[6] T. Vesa, P. Pochart and P. Marteau, "Pharmacokinetics of Lactobacillus plantarum NCIMB 8826, Lactobacillus fermentum KLD, and Lactococcus lactis MG1363 in the Human Gastrointestinal Tract," Alimentary Pharmacology \& Therapeutics, Vol. 14, No. 6, 2000, pp. 823-828. doi:10.1046/j.1365-2036.2000.00763.x

[7] C. de Champs, N. Maroncle, D. Balestrino, C. Rich and C. Forestier, "Persistence of colonization of Intestinal $\mathrm{Mu}$ cosa by a Probiotic Strain, Lactobacillus casei Subsp. rhamnosus LCR35, after Oral Consumption," Journal of Clinical Microbiology, Vol. 41, No. 3, 2003, pp. 12701273. doi:10.1128/JCM.41.3.1270-1273.2003

[8] N. Valeur, P. Engel, N. Carbajal, E. Connolly and K. Ladefoged, "Colonization and Immunomodulation by Lac- 
tobacillus reuteri ATCC 55730 in the Human Gastrointestinal Tract," Applied and Environmental Microbiology, Vol. 70, No. 2, 2004, pp. 1176-1181. doi:10.1128/AEM.70.2.1176-1181.2004

[9] T. D. Klingberg and B. B. Budde, "The Survival and Persistence in the Human Gastrointestinal Tract of Five Potential Probiotic Lactobacilli Consumed as FreezeDried Cultures or as Probiotic Sausage,” International Journal of Food Microbiology, Vol. 109, No. 1-2, 2006, pp. 157-159. doi:10.1016/j.ijfoodmicro.2006.01.014

[10] R. Oozeer, A. Leplingard, D. D. Mater, A. Mogenet, R. Michelin, I. Seksek, P. Marteau, J. Dore, J. L. Bresson and G. Corthier, "Survival of Lactobacillus casei in the Human Digestive Tract after Consumption of Fermented Milk," Applied and Environmental Microbiology, Vol. 72, No. 8, 2006, pp. 5615-5617. doi:10.1128/AEM.00722-06

[11] K. M. Tuohy, M. Pinart-Gilberga, M. Jones, L. Hoyles, A. L. McCartney and G. R. Gibson, "Survivability of a Probiotic Lactobacillus casei in the Gastrointestinal Tract of Healthy Human Volunteers and Its Impact on the Faecal Microflora,” Journal of Applied Microbiology, Vol. 102, No. 4, 2007, pp. 1026-1032.

[12] Y. E. Dommels, R. A. Kemperman, Y. E. Zebregs, R. B. Draaisma, A. Jol, D. A. Wolvers, E. E. Vaughan and R. Albers, "Survival of Lactobacillus reuteri DSM 17938 and Lactobacillus rhamnosus GG in the Human Gastrointestinal Tract with Daily Consumption of a Low-Fat Probiotic Spread,” Applied and Environmental Microbiology, Vol. 75, No. 19, 2009, pp. 6198-6204. doi:10.1128/AEM.01054-09

[13] B. Stecher and W. D. Hardt, "Mechanisms Controlling Pathogen Colonization of the Gut," Current Opinion in Microbiology, Vol. 14, No. 1, 2011, pp. 82-91. doi:10.1016/j.mib.2010.10.003

[14] J. Walter, "Ecological Role of Lactobacilli in the Gastrointestinal Tract: Implications for Fundamental and Biomedical Research," Applied and Environmental Microbiology, Vol. 74, 2008, pp. 4985-4996. doi:10.1128/AEM.00753-08

[15] G. W. Tannock, K. Munro, H. J. Harmsen, G. W. Welling, J. Smart and P. K. Gopal, "Analysis of the Fecal Microflora of Human Subjects Consuming a Probiotic Product Containing Lactobacillus rhamnosus DR20," Applied and Environmental Microbiology, Vol. 66, No. 6, 2000, pp. 2578-2588. doi:10.1128/AEM.66.6.2578-2588.2000

[16] G. Reuter, "The Lactobacillus and Bifidobacterium Microflora of the Human Intestine: Composition and Succession," Current Issues in Intestinal Microbiology, Vol. 2, No. 2, 2001, pp. 43-53.

[17] D. C. Savage, "Microbial Ecology of the Gastrointestinal Tract," Annual Review of Microbiology, Vol. 31, 1977, pp. 107-133. doi:10.1146/annurev.mi.31.100177.000543

[18] M. A. Azcarate-Peril, E. Altermann, Y. J. Goh, R. Tallon, R. B. Sanozky-Dawes, E. A. Pfeiler, S. O’Flaherty, B. L. Buck, A. Dobson, T. Duong, M. J. Miller, R. Barrangou and T. R. Klaenhammer, "Analysis of the Genome Sequence of Lactobacillus gasseri ATCC 33323 Reveals the Molecular Basis of an Autochthonous Intestinal Organism,” Applied and Environmental Microbiology, Vol. 74,
No. 15 , 2008, pp. 4610-4625. doi:10.1128/AEM.00054-08

[19] B. M. Forde, B. A. Neville, M. M. O’Donnell, E. Riboulet-Bisson, M. J. Claesson, A. Coghlan, R. P. Ross and P. W. O'Toole, "Genome Sequences and Comparative Genomics of Two Lactobacillus ruminis Strains from the Bovine and Human Intestinal Tracts," Microbial Cell Factories, Vol. 10, Suppl. 1, 2011, p. S13. doi:10.1186/1475-2859-10-S1-S13

[20] S. A. Frese, A. K. Benson, G. W. Tannock, D. M. Loach, J. Kim, M. Zhang, P. L. Oh, N. C. Heng, P. B. Patil, N. Juge, D. A. Mackenzie, B. M. Pearson, A. Lapidus, E. Dalin, H. Tice, E. Goltsman, M. Land, L. Hauser, N. Ivanova, N. C. Kyrpides and J. Walter, "The Evolution of Host Specialization in the Vertebrate Gut Symbiont Lactobacillus reuteri," PLoS Genet, Vol. 7, No. 2, 2011, Article ID: e1001314. doi:10.1371/journal.pgen.1001314

[21] P. L. Oh, A. K. Benson, D. A. Peterson, P. B. Patil, E. N. Moriyama, S. Roos and J. Walter, "Diversification of the Gut Symbiont Lactobacillus reuteri as a Result of HostDriven Evolution," The ISME Journal, Vol. 4, No. 3, 2010, pp. 377-387. doi:10.1038/ismej.2009.123

[22] S. Rabot, J. Rafter, G. T. Rijkers, B. Watzl and J. M. Antoine, "Guidance for Substantiating the Evidence for Beneficial Effects of Probiotics: Impact of Probiotics on Digestive System Metabolism,” Journal of Nutrition, Vol. 140, No. 3, 2010, pp. 677S-689S. doi:10.3945/jn.109.113738

[23] M. E. Sanders and T. R. Klaenhammer, "Invited Review: The Scientific Basis of Lactobacillus acidophilus NCFM Functionality as a Probiotic,” Journal of Dairy Science, Vol. 84, No. 2, 2001, pp. 319-331. doi:10.3168/jds.S0022-0302(01)74481-5

[24] J. Walter, C. Hertel, G. W. Tannock, C. M. Lis, K. Munro and W. P. Hammes, "Detection of Lactobacillus, Pediococcus, Leuconostoc, and Weissella Species in Human Feces by Using Group-Specific PCR Primers and Denaturing Gradient Gel Electrophoresis,” Applied and Environmental Microbiology, Vol. 67, No. 6, 2001, pp. 25782585. doi:10.1128/AEM.67.6.2578-2585.2001

[25] E. Wesney and G. W. Tannock, “Association of Rat, Pig, and Fowl Biotypes of Lactobacilli with the Stomach of Gnotobiotic Mice,” Microbial Ecology, Vol. 5, No. 1, 1979, pp. 35-42. doi:10.1007/BF02010576

[26] S. Roos, F. Karner, L. Axelsson and H. Jonsson, "Lactobacillus Mucosae sp. nov., a New Species with in Vitro Mucus-Binding Activity Isolated from Pig Intestine,” International Journal of Systematic and Evolutionary Microbiology, Vol. 50, No. 1, 2000, pp. 251-258. doi:10.1099/00207713-50-1-251

[27] L. Axelsson and S. Lindgren, "Characterization and DNA Homology of Lactobacillus Strains Isolated from Pig Intestine,” Journal of Applied Bacteriology, Vol. 62, No. 5, 1987, pp. 433-440. doi:10.1111/j.1365-2672.1987.tb02673.x

[28] C. Efthymiou and P. A. Hansen, “An Antigenic Analysis of Lactobacillus Acidophilus,” The Journal of Infectious Diseases, Vol. 110, No. 3, 1962, pp. 258-267. doi:10.1093/infdis/110.3.258 
[29] I. Martinez, J. Kim, P. R. Duffy, V. L. Schlegel and J. Walter, "Resistant Starches Types 2 and 4 Have Differential Effects on the Composition of the Fecal Microbiota in Human Subjects,” PLoS One, Vol. 5, No. 11, 2010, p. e15046. doi:10.1371/journal.pone.0015046

[30] P. Kabadjova, X. Dousset, V. Le Cam and H. Prevost, "Differentiation of Closely Related Carnobacterium Food Isolates Based on 16s-23s Ribosomal DNA Intergenic Spacer Region Polymorphism,” Applied and Environmental Microbiology, Vol. 68, No. 11, 2002, pp. 53585366. doi:10.1128/AEM.68.11.5358-5366.2002

[31] M. Haarman and J. Knol, "Quantitative Real-Time PCR Analysis of Fecal Lactobacillus Species in Infants Receiving a Prebiotic Infant Formula," Applied and Environmental Microbiology, Vol. 72, No. 4, 2006, pp. 23592365. doi:10.1128/AEM.72.4.2359-2365.2006

[32] C. B. Meroth, J. Walter, C. Hertel, M. J. Brandt and W. P. Hammes, "Monitoring the Bacterial Population Dynamics in Sourdough Fermentation Processes by Using PCR-Denaturing Gradient Gel Electrophoresis,” Applied and Environmental Microbiology, Vol. 69, No. 1, 2003, pp. 475482. doi:10.1128/AEM.69.1.475-482.2003

[33] S. Hammons, P. L. Oh, I. Martinez, K. Clark, V. L. Schlegel, E. Sitorius, S. E. Scheideler and J. Walter, “A Small Variation in Diet Influences the Lactobacillus Strain Composition in the Crop of Broiler Chickens," Systematic and Applied Microbiology, Vol. 33, No. 5, 2010, pp. 275-281. doi:10.1016/j.syapm.2010.04.003

[34] B. Dal, F. Walter, W. J. Hammes and C. Hertel, "Increased Complexity of the Species Composition of Lactic Acid Bacteria in Human Feces Revealed by Alternative Incubation Condition,” Microbial Ecology, Vol. 45, No. 4, 2003, pp. 455-463. doi:10.1007/s00248-003-2001-z

[35] J. Sui, S. Leighton, F. Busta and L. Brady, “16s Ribo-

\section{Abbreviations and Acronyms}

GI: gastrointestinal;

RAPD: random amplification of polymorphic DNA; qPCR: quantitative real-time PCR;

PCR: polymerase chain reaction; somal DNA Analysis of the Faecal Lactobacilli Composition of Human Subjects Consuming a Probiotic Strain Lactobacillus acidophilus NCFM," Journal of Applied Microbiology, Vol. 93, No. 5, 2002, pp. 907-912. doi:10.1046/j.1365-2672.2002.01767.x

[36] M. Watanabe, H. Kinoshita, M. Nitta, R. Yukishita, Y. Kawai, K. Kimura, N. Taketomo, Y. Yamazaki, Y. Tateno, K. Miura, A. Horii, H. Kitazawa and T. Saito, "Identification of a New Adhesin-Like Protein from Lactobacillus mucosae ME-340 with Specific Affinity to the Human Blood Group a and b Antigens," Journal of Applied Microbiology, Vol. 109, No. 3, 2010, pp. 927-935. doi:10.1111/j.1365-2672.2010.04719.x

[37] L. Dethlefsen, P. B. Eckburg, E. M. Bik and D. A. Relman, "Assembly of the Human Intestinal Microbiota," Trends in Ecology \& Evolution, Vol. 21, No. 9, 2006, pp. 517-523. doi:10.1016/j.tree.2006.06.013

[38] J. Cavender-Bares, K. H. Kozak, P. V. Fine and S. W. Kembel, "The Merging of Community Ecology and Phylogenetic Biology,” Ecology Letters, Vol. 12, No. 7, 2009, pp. 693-715. doi:10.1111/j.1461-0248.2009.01314.X

[39] J. Walter and R. Ley, "The Human Gut Microbiome: Ecology and Recent Evolutionary Changes,” Annual Review of Microbiology, Vol. 65, No. 10, 2011, pp. 411429. doi:10.1146/annurev-micro-090110-102830

[40] A. Khoruts, J. Dicksved, J. K. Jansson and M. J. Sadowsky, "Changes in the Composition of the Human Fecal Microbiome after Bacteriotherapy for Recurrent Clostridium difficile-Associated Diarrhea,” Journal of Clinical Gastroenterology, Vol. 44, No. 5, 2010, pp. 354-360.

[41] J. O’Callaghan and P. W. O’Toole, "Lactobacillus: HostMicrobe Relationships,” Current Topics in Microbiology and Immunology, in Press, 2012.

MRS: mann-rogosa-sharpe media;

NCBI: national center for biotechnology information;

MLSA: multi-locus sequencing analysis;

IRB: institutional review board;

CFU: colony-forming units. 